\title{
Nitrogênio em cobertura e estresse salino na qualidade fisiológica de sementes de meio-irmãos de milho, no Tocantins
}

\author{
Top dressed nitrogen and salt stress in seed physiology quality of half-sib maize families in \\ Tocantins
}

\author{
Edmar Vinicius de Carvalho ${ }^{1 *}$, Flávio Sérgio Afférri ${ }^{2}$, Eliane Aparecida Rotili ${ }^{1}$, Ana Luísa Lopes \\ Ernesto Reis ${ }^{1}$, Greice da Silva Ribeiro³, Karen Cristina Leite Silva1 \& Joênes Mucci Peluzio1 \\ ${ }^{1}$ Universidade Federal do Tocantins, Gurupi, TO, Brasil. *Autor para correspondência: carvalho.ev@uft.edu.br. \\ 2 Universidade Federal de São Carlos, Buri, SP, Brasil. \\ ${ }^{3}$ Naturatins, Araguaína, TO, Brasil.
}

Submissão: 24/09/2015 / Aceite: 09/05/2018

\begin{abstract}
RESUMO
Na cultura do milho, o genótipo e a adubação afetam a produção de sementes de qualidade, e o uso de testes de vigor em condições de estresse permite identificar genótipos superiores. Objetivou-se, neste trabalho, verificar o efeito da adubação nitrogenada em cobertura na qualidade fisiológica de sementes de 12 meio-irmãos de milho, com ou sem estresse salino. Essas sementes foram colhidas de plantas submetidas a duas doses de $\mathrm{N}$ em cobertura $\left(0 ; 150 \mathrm{~kg} \mathrm{ha}^{-1}\right)$, na safra 2012/2013, de Gurupi, TO. Em laboratório, foram conduzidos dois experimentos: sem e com estresse salino $\left(\mathrm{KCl}-16,73 \mathrm{~g} \mathrm{~L}^{-1}\right)$. O delineamento utilizado foi inteiramente ao acaso, com esquema fatorial $(12 \times 2 \times 2)$ e quatro repetições. Os seguintes testes foram realizados: germinação, comprimento e massa seca de plântulas. Os efeitos significativos do $\mathrm{N}$ em cobertura foram observados em maior grau quando em condição de germinação com estresse salino. Nessa condição, quando as sementes foram obtidas sem uso de $\mathrm{N}$ em cobertura, 0 meio-irmão L8 apresentou médias superiores de comprimento total $(15,3 \mathrm{~cm})$, radicular $(9,54 \mathrm{~cm})$ e da parte aérea $(5,75 \mathrm{~cm})$, e de massa seca radicular $\left(26 \mathrm{mg}\right.$ plântula $\left.\mathrm{a}^{-1}\right)$ e da parte aérea (28 $\left.\mathrm{mg} \mathrm{plântula}^{-1}\right)$. 0 meio-irmão L9 apresentou influência significativa (positiva) do $\mathrm{N}$ nos atributos avaliados, quando a germinação foi realizada com estresse salino. A concentração de $16,73 \mathrm{~g} \mathrm{~L}^{-1}$ de $\mathrm{KCl}$ promoveu reduções significativas na germinação e vigor das sementes, porém, permitiu a visualização do efeito da adubação de $\mathrm{N}$ em cobertura. Em condições sem uso do $\mathrm{N}$ em cobertura e estresse salino na germinação, destacaram-se os meio-irmãos P6 e L8.
\end{abstract}

PALAVRAS-CHAVE: Cerrado, estresse mineral, germinação, vigor, Zea mays L.

\section{ABSTRACT}

Genotype and plant nutrition influence the production of physiological qualities in maize seeds, and the use of vigor tests under stress conditions allows for the identification of superior genotypes. The aim of this study was to verify the effect of covered nitrogen fertilization on the physiological quality of seeds from 12 half-sib maize families, with or without saline stress. Seeds were used from plants that were growing with two levels of top dressed nitrogen fertilization $\left(0 ; 150 \mathrm{~kg} \mathrm{ha}^{-1}\right)$, in the 2012/13 growing season, at Gurupi, in the state of Tocantins. In the laboratory, two experiments were conducted: one without and another with saline stress $\left(\mathrm{KCl}-16.73 \mathrm{~g} \mathrm{~L}^{-1}\right)$. The experimental design was fully randomized, with a factorial arrangement $(12 \times 2 \times 2)$ and four replications. The following tests were carried out: germination, length and dry weight of seedling. The effects of topdressed nitrogen were more visible in the conditions of saline stress on the seeds. In this situation, when seeds were produced without topdressed $\mathrm{N}$ the half-sib L8 showed higher: total length $(15.3 \mathrm{~cm})$, root length $(9.54 \mathrm{~cm})$ and aerial part length $(5.75 \mathrm{~cm})$, and dry weight of roots $\left(26 \mathrm{mg} \mathrm{seedling}^{-1}\right)$ and aerial part $\left(28 \mathrm{mg}\right.$ seedling $\left.{ }^{-1}\right)$. The half-sib L9 had shown significant (positive) influence from the use of topdressed $\mathrm{N}$ when germination was carried out with saline stress. The concentration of $\mathrm{KCl}\left(16.73 \mathrm{~g} \mathrm{~L}^{-1}\right)$ promoted significant decreases in the germination and vigor of the seeds, however, it allowed for the visualization of the effect of covered nitrogen fertilization. In conditions without topdressed $\mathrm{N}$ and saline stress at germination, the half-sibs P6 and L8 showed superiority.

KEYWORDS: Cerrado, germination, mineral stress, vigor, Zea mays L. 


\section{INTRODUÇÃO}

A baixa produtividade do milho em algumas regiões possui relação com a baixa fertilidade dos solos (PEREIRA et al. 2015) e com a falta de sementes melhoradas de variedades que sejam adaptadas às condições locais (CARVALHO \& SOUZA 2007). Além dos atributos de interesse, em um programa de melhoramento vegetal é importante avaliar a qualidade fisiológica da semente (OLIVEIRA et al. 2013), pois ela contém a tecnologia oriunda do trabalho de seleção (PEREIRA et al. 2015). Essa qualidade é adquirida no desenvolvimento da semente e, posteriormente, perdida ou diminuída, quando as condições de armazenamento/germinação não são ideais (ABREU et al. 2014).

A produção de sementes de qualidade deve ser realizada em condições favoráveis, por exemplo, fornecendo os nutrientes exigidos pela cultura na forma de adubação (OLIVEIRA et al. 2013), em que o nitrogênio $(\mathrm{N})$ é o nutriente mais requerido e mais capaz de limitar a produção (ZUCARELI et al. 2012, PEREIRA et al. 2015). Com relação à formação de sementes, o nitrogênio presente nos órgãos vegetativos é translocado para essas estruturas, durante a fase reprodutiva (DUETE et al. 2008), na forma de aminoácidos e proteínas (MARSCHNER 1995). Componentes estes que são fundamentais para a germinação e que se relacionam diretamente à qualidade fisiológica da semente (FERREIRA et al. 2001).

No entanto, quando as sementes são submetidas a condições de estresse, a visualização de diferenças relacionadas à qualidade fisiológica é facilitada, em virtude da maior expressão da diversidade genética nessas condições (NERLING et al. 2013).

$\mathrm{Na}$ avaliação do vigor de sementes podem ser utilizadas condições de estresse como, por exemplo, o estresse hídrico e térmico (GRZYBOWSKI et al. 2015, SBRUSSI \& ZUCARELI 2015) ou o salino. Nessas condições, ocorre menor absorção de água, decorrente da redução do potencial osmótico, que aumenta as forças de retenção da água pelo meio/substrato (MOTERLE et al. 2006), e de nutrientes, o que promove diminuição do fluxo das reservas do endosperma para o eixo embrionário (TONEL et al. 2013).

O vigor é caracterizado como um conjunto de atributos distintos (SBRUSSI \& ZUCARELI 2015) que se manifestam na habilidade de um organismo germinar num amplo limite de condições (ÁVILA et al. 2007). O genótipo exerce influência na qualidade fisiológica da semente (NERLING et al. 2014), e por meio dela, pode-se fazer a seleção dos superiores (ABREU et al. 2014), com a possibilidade de obter ganhos genéticos (NERLING et al. 2013). Essa diferença entre cultivares/genótipos pode ser visualizada por meio de testes de vigor ou pela mensuração da atividade enzimática (TIMÓTEO \& MARCOS FILHO 2013).

Variedades e/ou populações de polinização aberta possuem importância sócioeconômica (NERLING et al. 2014) e base genética ampla, que conferem maior plasticidade (ALLARD \& BRADSHAW 1964) e, assim, maior adaptação a condições de estresse (CHIMONYO et al. 2014), o que é uma vantagem para germinação em condições desfavoráveis (NERLING et al. 2013). Essa grande variabilidade, desejável para o melhoramento, é encontrada em progênies de meio-irmãos de milho, como relatam os trabalhos de CARVALHO et al. (2000) e COLOMBO et al. (2014).

Diante do exposto, o objetivo do trabalho foi verificar o efeito da adubação nitrogenada em cobertura na qualidade fisiológica de sementes, sob condições de estresse salino, de 12 meio-irmãos de milho produzidas no Tocantins.

\section{MATERIAL E MÉTODOS}

Foram utilizadas sementes de 12 meio-irmãos de milho, oriundos de seis populações de polinização aberta (P1, P2, P3, P4, P5 e P6) e de seis linhagens $S_{6}(L 1, L 2, L 3, L 4, L 5$ e L6). As sementes foram obtidas de plantas submetidas a duas doses de nitrogênio em cobertura (0 e $150 \mathrm{~kg} \mathrm{ha}^{-1}$ de $\mathrm{N}$, dose segundo recomendação de RIBEIRO et al. (1999), obtida pela interpretação da análise de solo), cultivadas na safra verão 2012/2013, no município de Gurupi, TO. A adubação de cobertura foi realizada 27 dias após a semeadura (entre os estágios V4-V6), e a colheita das espigas, após a maturação fisiológica (estágio R6). As espigas foram debulhadas manualmente, e as sementes, limpas e armazenadas em local com controle de temperatura e umidade, até o momento das análises em laboratório.

Foram conduzidos dois experimentos em laboratório: um sem estresse salino (condições normais) e o outro com estresse salino, com uso de solução de cloreto de potássio $(\mathrm{KCl})$ na concentração de $16,73 \mathrm{~g} \mathrm{~L}^{-1}$ para obtenção de potencial osmótico superior a $-0,9 \mathrm{MPa}$, de acordo com a fórmula de van't Hoff (MOTERLE et al. 2006). As sementes utilizadas foram obtidas de 12 meio-irmãos de milho provenientes de cultivos com e sem adubação de nitrogênio em cobertura.

$\mathrm{Na}$ avaliação da qualidade fisiológica, as sementes foram submetidas às seguintes determinações: Germinação: conduzido com quatro repetições de 50 sementes, em papel toalha umedecido na proporção de 2,5 vezes a massa (g) do substrato (água destilada ou solução com $\mathrm{KCl}$ na concentração de 16,73 $\mathrm{g} \mathrm{L}^{-1}$ ). 
Os rolos de papel foram mantidos em germinador tipo câmara, na posição horizontal, sob temperatura de $30{ }^{\circ} \mathrm{C}$. As contagens foram realizadas durante quatro dias após a instalação do teste (primeira contagem) e, posteriormente, durante sete dias adicionais (contagem final), seguindo os critérios estabelecidos em BRASIL (2009). Os resultados foram expressos em porcentagem.

Comprimento de plântulas: após a avaliação das plântulas normais, na última contagem do teste de germinação, mediu-se o comprimento de 10 plântulas em cada repetição. O comprimento médio das plântulas (parte aérea, radicular e total) foi obtido somando-se as medidas de cada medição e dividindo-se o resultante pelo número de plântulas normais mensuradas, com resultados expressos em centímetros (DIAS \& BARROS 1995).

Massa seca de plântulas: após a avaliação das plântulas normais, na última contagem do teste de germinação, estas foram separadas em duas porções: parte aérea e radicular. As porções foram acondicionadas em sacos de papel, levadas à estufa a $60 \pm 5{ }^{\circ} \mathrm{C}$ até atingirem massa constante e, após, pesadas em balança com precisão de $0,001 \mathrm{~g}$, obtendo-se a massa seca. Todos os valores foram divididos pelo número de plântulas avaliadas (10), sendo expressos os resultados em miligramas por plântula de cada porção (NAKAGAWA 1999).

Os dados foram submetidos à análise de variância, considerando o delineamento inteiramente ao acaso no esquema fatorial: 2 (com e sem estresse salino) x 12 (meio-irmãos) $\times 2$ (0 e $150 \mathrm{~kg} \mathrm{ha}^{-1} \mathrm{de} \mathrm{N} \mathrm{em}$ cobertura), com quatro repetições. Foram aplicados o teste de Scott-Knott $(p<0,05)$, na comparação das médias dos meio-irmãos dentro de cada nível de $\mathrm{N}$, e o teste Tukey $(p<0,05)$, na comparação da resposta de cada genótipo com uso do $\mathrm{N}$ em cobertura. Por fim, as médias obtidas em cada experimento (com estresse salino; sem estresse salino) foram comparadas entre si pelo teste $t(p<0,05)$.

\section{RESULTADOS E DISCUSSÃO}

Foi detectado efeito significativo, pela análise de variância $(p<0,05)$, do estresse salino de maneira isolada na análise de variância e na interação tripla entre meio-irmãos, dose de nitrogênio em cobertura e estresse salino (dados não mostrados). Em virtude destes resultados, foi realizado o desdobramento dentro da cada concentração de $\mathrm{KCl}\left(0,00 \mathrm{~g} \mathrm{~L}^{-1}\right.$ ou $\left.16,73 \mathrm{~g} \mathrm{~L}^{-1}\right)$ para avaliar a diferença e resposta dos meio-irmãos nas duas doses de $\mathrm{N}$ em cobertura ( 0 ou $150 \mathrm{~kg} \mathrm{ha}^{-1}$ ).

Os resultados do desdobramento da análise de variância (Tabela 1) indicam viabilidade de identificação de genótipos superiores, quanto à qualidade fisiológica da semente, em condições de germinação com e sem estresse salino. ROTILI et al. (2015) avaliaram os genótipos que deram origem aos meio-irmãos e encontraram divergência genética em condições com e sem fornecimento de $\mathrm{N}$, com a formação de até nove grupos pelo método de Tocher. Assim, é possível destacar a existência da variabilidade genética entre os meio-irmãos avaliados, a qual é essencial para o melhoramento.

Tabela 1. Resumo dos resultados do desdobramento da análise de variância, em cada condição de estresse salino, da qualidade fisiológica de sementes de 12 meio-irmãos (MI) de milho cultivados com dois níveis de nitrogênio $(\mathrm{N})$ em cobertura $\left(0 ; 150 \mathrm{~kg} \mathrm{ha}^{-1}\right)$, Tocantins, 2012/2013.

Table 1. Summary of variance analysis deployment, in each condition of saline stress, of physiological quality seed of 12-maize half-sib families growing with two levels of covered nitrogen $(0 ; 150 \mathrm{~kg} \mathrm{ha}$ 1), Tocantins, 2012/2013.

\begin{tabular}{|c|c|c|c|c|c|c|c|c|c|c|}
\hline \multirow{2}{*}{$\begin{array}{l}\text { Fonte de } \\
\text { Variação }\end{array}$} & \multicolumn{5}{|c|}{ Sem estresse salino } & \multicolumn{5}{|c|}{ Com estresse salino } \\
\hline & $\mathrm{Ml}$ & $\mathrm{N}$ & $\mathrm{MI}^{*} \mathrm{~N}$ & $\mathrm{CV}$ & Média & $\mathrm{Ml}$ & $\mathrm{N}$ & $\mathrm{MI}^{*} \mathrm{~N}$ & $\mathrm{CV}$ & Média \\
\hline $\mathrm{G}_{4}$ & & ns & ns & 1,48 & $99,0^{a}$ & $\overline{* \star}$ & ns & ns & 3,44 & $96,0^{b}$ \\
\hline $\mathrm{G}_{7}$ & * & ns & ns & 1,17 & $99,0^{a}$ & * & ns & ns & 2,65 & $98,0^{b}$ \\
\hline $\mathrm{C}_{\mathrm{T}}$ & ** & ns & ** & 8,96 & $27,62^{a}$ & ** & ** & ** & 12,44 & $12,89^{b}$ \\
\hline $\mathrm{C}_{\mathrm{R}}$ & ** & ns & ns & 13,45 & $14,89^{a}$ & ** & $* *$ & ** & 13,68 & 8,24 b \\
\hline $\mathrm{CPA}_{\mathrm{PA}}$ & ** & ns & ** & 9,08 & $12,74^{a}$ & $* *$ & $* *$ & ** & 14,16 & $4,65^{b}$ \\
\hline$M S_{R}$ & ** & ns & ns & 16,39 & $30,64^{a}$ & $\star \star *$ & $\star *$ & ** & 19,48 & $18,73^{b}$ \\
\hline$M S_{P A}$ & ** & ns & ns & 9,82 & $38,92^{a}$ & $* *$ & ns & ** & 24,93 & $19,88^{b}$ \\
\hline
\end{tabular}

$\mathrm{G}_{4}, \mathrm{G}_{7}=$ percentagem de germinação na primeira e na última contagem; $\mathrm{C}_{\mathrm{T}}=$ comprimento total $(\mathrm{cm}) ; \mathrm{C}_{\mathrm{R}}=$ comprimento radicular (cm); $\mathrm{C}_{\mathrm{PA}}=$ comprimento da parte aérea $(\mathrm{cm}) ; \mathrm{MS}_{\mathrm{R}}=$ massa seca radicular $\left(\mathrm{mg}\right.$ plântula $\left.{ }^{-1}\right) ; \mathrm{MS}_{\mathrm{PA}}=$ massa seca da parte aérea (mg plântula $\left.{ }^{1}\right) ;{ }^{*},{ }^{* *}=$ significativo pelo teste $F\left(p<0,05 ; p<0,01\right.$; respectivamente). ${ }^{n s}=$ não significativo pelo teste $F(p>0,05)$. Médias seguidas de mesmas letras (expoentes) na linha, não apresentam diferença significativa pelo teste $t(p>0,05)$.

O estresse salino influenciou de forma significativa $(p<0,05)$ e negativa as médias dos atributos avaliados (Tabela 1), em que as pequenas diferenças consideradas como significativas, relacionadas à 
germinação, têm relação com o baixo coeficiente de variação (PEREIRA et al. 2015).

ÁVILA et al. (2007) observaram redução do comprimento radicular, com redução do potencial osmótico, e MOTERLE et al. (2006) encontraram reduções na germinação, comprimento e massa seca das plântulas, com aumento da concentração de KCl. TONEL et al. (2013) verificaram redução do comprimento e da massa seca das plântulas, com aumento da concentração de $\mathrm{NaCl}$, aliada a menor atividade das enzimas SOD, APX e CAT, e, por fim, CONUS et al. (2009) constataram redução do comprimento de plântulas sob maiores concentrações de $\mathrm{KCl}$.

Nas condições de estresse salino, ocorre menor absorção de água e nutrientes e, em consequência, diminuição do fluxo das reservas do endosperma para o eixo embrionário (TONEL et al. 2013). Essa menor absorção tem relação com a redução do potencial osmótico, que aumenta as forças de retenção da água pelo meio/substrato (MOTERLE et al. 2006). A redução do crescimento está envolvida com a diminuição da expansão celular sob condições de estresse, como por exemplo, o estresse salino (TAIZ \& ZEIGER 2009).

Os efeitos significativos do nitrogênio em cobertura, de maneira isolada ou em interação com os meio-irmãos, foram observados em maior grau quando as sementes foram colocadas para germinar em condições de estresse salino (Tabela 1). Os atributos que apresentaram influências significativas $(p<0,05)$ foram os relacionados ao vigor da semente (comprimento e massa seca das partes da plântula).

ZUCARELI et al. (2012), na avaliação de variedades de milho doce, observaram que a resposta dos genótipos foi diferente em função da dose de N, no entanto, sem influência do N na germinação e na massa seca das partes das plântulas, em condições sem indução de estresses. Nesse sentido, a indução de estresse permite que seja visualizado o efeito do $\mathrm{N}$ na qualidade fisiológica de sementes, concordando com as afirmações de GRZYBOWSKI et al. (2015) e SBRUSSI \& ZUCARELI (2015).

A germinação das sementes, tanto na primeira contagem quanto na última, apresentou resultado acima de $90 \%$ (Tabela 2), superior ao mínimo descrito para comercialização pela legislação brasileira em vigor (Lei no 10.711/2003). Dos meio-irmãos avaliados, seis foram classificados no grupo estatístico superior nas duas condições de germinação, independentemente da dose de $\mathrm{N}$ utilizada para obtenção das sementes, com valores $\geq 96 \%$. De acordo com GRZYBOWSKI et al. (2015), sementes com germinação entre 96 e 100\% podem ser classificadas como de alta qualidade, e segundo CONUS et al. (2009) a adaptação ao estresse pode ser detectada pela germinação e crescimento nessas condições.

Tabela 2. Percentagem de germinação na primeira e última contagem $\left(\mathrm{G}_{4}, \mathrm{G}_{7}\right.$, em \%), de 12 meio-irmãos de milho cultivados com dois níveis de nitrogênio em cobertura $\left(0 ; 150 \mathrm{~kg} \mathrm{ha}^{-1}\right)$, em condições de germinação com ou sem estresse salino. Tocantins, 2012/2013.

Table 2. Germination percentage of the first and last count $\left(G_{4}, G_{7}\right.$, in \%), of the 12-maize half-sib families growing with two levels of covered nitrogen $\left(0 ; 150 \mathrm{~kg} \mathrm{ha}^{-1}\right)$, in germination conditions with and without saline stress. Tocantins, 2012/2013.

\begin{tabular}{|c|c|c|c|c|c|c|c|c|c|c|c|c|c|c|c|c|}
\hline \multirow{4}{*}{$\begin{array}{l}\text { Meio- } \\
\text { irmãos }\end{array}$} & \multicolumn{8}{|c|}{ Com estresse salino } & \multicolumn{8}{|c|}{ Sem estresse salino } \\
\hline & \multicolumn{4}{|c|}{$\mathrm{G}_{4}$} & \multicolumn{4}{|c|}{$\mathrm{G}_{7}$} & \multicolumn{4}{|c|}{$\mathrm{G}_{4}$} & \multicolumn{4}{|c|}{$\mathrm{G}_{7}$} \\
\hline & \multicolumn{2}{|c|}{ ON } & \multicolumn{2}{|c|}{$150 \mathrm{~N}$} & \multicolumn{2}{|c|}{ ON } & \multicolumn{2}{|c|}{$150 \mathrm{~N}$} & \multicolumn{2}{|c|}{ ON } & \multicolumn{2}{|c|}{$150 \mathrm{~N}$} & \multicolumn{2}{|c|}{ ON } & \multicolumn{2}{|c|}{$150 \mathrm{~N}$} \\
\hline & 98 & $\mathrm{Aa}$ & 99 & $\mathrm{Aa}$ & 99 & $\mathrm{Aa}$ & 99 & $\mathrm{Aa}$ & 100 & $\mathrm{Aa}$ & 100 & $\mathrm{Aa}$ & 100 & $\mathrm{Aa}$ & 100 & $\mathrm{Aa}$ \\
\hline P2 & 96 & $\mathrm{Aa}$ & 98 & $\mathrm{Aa}$ & 97 & $\mathrm{Aa}$ & 98 & $A_{a}$ & 100 & $\mathrm{Aa}$ & 100 & $\mathrm{Aa}$ & 100 & $\mathrm{Aa}$ & 100 & $\mathrm{Aa}$ \\
\hline P3 & 97 & $\mathrm{Aa}$ & 97 & $\mathrm{Aa}$ & 98 & $\mathrm{Aa}$ & 98 & $\mathrm{Aa}$ & 100 & $\mathrm{Aa}$ & 100 & $\mathrm{Aa}$ & 100 & $\mathrm{Aa}$ & 100 & $\mathrm{Aa}$ \\
\hline P4 & 98 & $\mathrm{Aa}$ & 97 & $\mathrm{Aa}$ & 99 & $\mathrm{Aa}$ & 98 & $\mathrm{Aa}$ & 98 & $\mathrm{Bb}$ & 100 & $\mathrm{Aa}$ & 98 & $\mathrm{Bb}$ & 100 & $\mathrm{Aa}$ \\
\hline P5 & 93 & $\mathrm{Ba}$ & 93 & $\mathrm{Ba}$ & 95 & $\mathrm{Aa}$ & 95 & $\mathrm{Aa}$ & 97 & $\mathrm{Ba}$ & 99 & $\mathrm{Aa}$ & 99 & $\mathrm{Ba}$ & 99 & $\mathrm{Ba}$ \\
\hline P6 & 98 & $\mathrm{Aa}$ & 98 & $\mathrm{Aa}$ & 98 & $\mathrm{Aa}$ & 98 & $\mathrm{Aa}$ & 100 & $\mathrm{Aa}$ & 100 & $\mathrm{Aa}$ & 100 & $\mathrm{Aa}$ & 100 & $\mathrm{Aa}$ \\
\hline L7 & 94 & $\mathrm{Ba}$ & 98 & $\mathrm{Aa}$ & 97 & $\mathrm{Aa}$ & 100 & $\mathrm{Aa}$ & 100 & $\mathrm{Aa}$ & 99 & $\mathrm{Aa}$ & 100 & $\mathrm{Aa}$ & 99 & $\mathrm{Ba}$ \\
\hline L8 & 98 & $\mathrm{Aa}$ & 99 & $\mathrm{Aa}$ & 99 & $\mathrm{Aa}$ & 99 & A & 100 & $\mathrm{Aa}$ & 100 & $\mathrm{Aa}$ & 100 & $\mathrm{Aa}$ & 100 & $\mathrm{Aa}$ \\
\hline L9 & 95 & $\mathrm{Ba}$ & 99 & $\mathrm{Aa}$ & 97 & $\mathrm{Aa}$ & 99 & $\mathrm{Aa}$ & 98 & $\mathrm{Ba}$ & 100 & $\mathrm{Aa}$ & 98 & $\mathrm{Ba}$ & 100 & $\mathrm{Aa}$ \\
\hline L10 & 95 & $\mathrm{Ba}$ & 93 & $\mathrm{Ba}$ & 97 & $\mathrm{Aa}$ & 97 & $\mathrm{Aa}$ & 100 & $\mathrm{Aa}$ & 99 & $\mathrm{Aa}$ & 100 & $\mathrm{Aa}$ & 99 & $\mathrm{Ba}$ \\
\hline L11 & 90 & $\mathrm{Ba}$ & 91 & $\mathrm{Ba}$ & 93 & $A b$ & 97 & $\mathrm{Aa}$ & 99 & $\mathrm{Aa}$ & 99 & $\mathrm{Aa}$ & 99 & $\mathrm{Aa}$ & 100 & $\mathrm{Aa}$ \\
\hline L12 & 95 & $\mathrm{Ba}$ & 94 & $\mathrm{Ba}$ & 98 & $\mathrm{Aa}$ & 98 & $\mathrm{Aa}$ & 98 & $\mathrm{Ba}$ & 98 & $\mathrm{Aa}$ & 98 & $\mathrm{Ba}$ & 98 & $\mathrm{Ba}$ \\
\hline
\end{tabular}

Médias com mesma letra pertencem ao mesmo grupo estatístico, na coluna pelo teste Scott-Knott $(p>0,05)$ e na linha pelo teste Tukey $(p>0,05)$.

Os meio-irmãos P4 e P5 foram os únicos com médias no grupo superior estatístico quanto ao comprimento médio radicular e à massa seca radicular, quando a condição de germinação foi sem estresse 
salino e independente da dose de $\mathrm{N}$ em cobertura usada no cultivo (Tabelas 3 e 5). O meio-irmão P6, além de média de massa seca radicular superior, também apresentou massa seca da parte área no grupo superior (Tabela 5).

Tabela 3. Comprimento médio total, radicular e da parte aérea $\left(C_{T}, C_{R}, C_{P A}\right.$, em $\left.\mathrm{cm}\right)$ das plântulas de 12 meio-irmãos de milho cultivados com dois níveis de nitrogênio em cobertura $\left(0 ; 150 \mathrm{~kg} \mathrm{ha}^{-1}\right)$, em condições de germinação sem estresse salino. Tocantins, 2012/2013.

Table 3. Total, radicular and aerial part length $\left(\mathrm{C}_{\mathrm{T}}, \mathrm{C}_{\mathrm{R}}, \mathrm{C}_{\mathrm{PA}}\right.$, in $\left.\mathrm{cm}\right)$ of seedlings of the 12-maize half-sib families growing with two levels of covered nitrogen $\left(0 ; 150 \mathrm{~kg} \mathrm{ha}^{-1}\right)$, in germination conditions without saline stress. Tocantins, 2012/2013.

\begin{tabular}{|c|c|c|c|c|c|c|c|c|c|c|c|c|}
\hline \multirow[t]{2}{*}{$\begin{array}{l}\text { Meio- } \\
\text { irmãos }\end{array}$} & \multicolumn{4}{|c|}{$\mathrm{C}_{\mathrm{T}}$} & \multicolumn{4}{|c|}{$C_{R}$} & \multicolumn{4}{|c|}{$\mathrm{CPA}_{\mathrm{PA}}$} \\
\hline & \multicolumn{2}{|c|}{$0 \mathrm{~N}$} & \multicolumn{2}{|c|}{$150 \mathrm{~N}$} & \multicolumn{2}{|r|}{$0 \mathrm{~N}$} & \multicolumn{2}{|r|}{$150 \mathrm{~N}$} & \multicolumn{2}{|r|}{$0 \mathrm{~N}$} & \multicolumn{2}{|r|}{$150 \mathrm{~N}$} \\
\hline P1 & 22,8 & $\mathrm{Ba}$ & 25,4 & $\mathrm{Ba}$ & 12,3 & $\mathrm{Ba}$ & 14,7 & $\mathrm{Aa}$ & 10,2 & $\mathrm{Ba}$ & 10,7 & $\mathrm{Da}$ \\
\hline P2 & 29,8 & $\mathrm{Aa}$ & 29,8 & $\mathrm{Aa}$ & 15,7 & $\mathrm{Aa}$ & 15,3 & $\mathrm{Aa}$ & 14,1 & $\mathrm{Aa}$ & 14,4 & $\mathrm{Ba}$ \\
\hline P3 & 28,9 & $\mathrm{Aa}$ & 28,3 & $\mathrm{Aa}$ & 16,7 & $\mathrm{Aa}$ & 15,9 & $\mathrm{Aa}$ & 12,2 & $\mathrm{Ba}$ & 12,4 & $\mathrm{Ca}$ \\
\hline P4 & 27,1 & $\mathrm{Aa}$ & 27,4 & $\mathrm{Ba}$ & 15,6 & $\mathrm{Aa}$ & 15,8 & $\mathrm{Aa}$ & 11,5 & $\mathrm{Ba}$ & 11,6 & $\mathrm{Da}$ \\
\hline P5 & 29,3 & $\mathrm{Aa}$ & 31,5 & $\mathrm{Aa}$ & 16,7 & $\mathrm{Aa}$ & 17,9 & $\mathrm{Aa}$ & 12,6 & $\mathrm{Ba}$ & 13,5 & $\mathrm{Ba}$ \\
\hline P6 & 28,0 & $\mathrm{Aa}$ & 24,4 & $\mathrm{Bb}$ & 15,7 & $\mathrm{Aa}$ & 12,6 & $A b$ & 12,7 & $\mathrm{Ba}$ & 11,7 & $\mathrm{Da}$ \\
\hline L7 & 27,1 & $\mathrm{Aa}$ & 25,6 & $\mathrm{Ba}$ & 13,8 & $\mathrm{Ba}$ & 14,7 & $\mathrm{Aa}$ & 13,3 & $\mathrm{Aa}$ & 10,9 & $\mathrm{Db}$ \\
\hline L8 & 23,3 & $\mathrm{Bb}$ & 30,7 & $\mathrm{Aa}$ & 11,7 & $\mathrm{Bb}$ & 14,8 & $\mathrm{Aa}$ & 11,6 & $\mathrm{Bb}$ & 15,8 & $\mathrm{Aa}$ \\
\hline L9 & 29,5 & $\mathrm{Aa}$ & 27,5 & $\mathrm{Ba}$ & 15,9 & $\mathrm{Aa}$ & 15,1 & $\mathrm{Aa}$ & 13,6 & $\mathrm{Aa}$ & 12,4 & $\mathrm{Ca}$ \\
\hline L10 & 25,6 & $\mathrm{Bb}$ & 29,6 & $\mathrm{Aa}$ & 12,2 & $\mathrm{Bb}$ & 15,4 & $\mathrm{Aa}$ & 13,4 & $\mathrm{Aa}$ & 14,2 & $\mathrm{Ba}$ \\
\hline L11 & 30,8 & $\mathrm{Aa}$ & 28,2 & $\mathrm{Aa}$ & 15,6 & $\mathrm{Aa}$ & 15,2 & $\mathrm{Aa}$ & 15,1 & $\mathrm{Aa}$ & 13,0 & $\mathrm{Cb}$ \\
\hline L12 & 26,3 & $\mathrm{Ba}$ & 26,5 & $\mathrm{Ba}$ & 14,1 & $\mathrm{Ba}$ & 13,8 & $\mathrm{Aa}$ & 12,3 & $\mathrm{Ba}$ & 12,7 & $\mathrm{Ca}$ \\
\hline
\end{tabular}

Médias com mesma letra pertencem ao mesmo grupo estatístico, na coluna pelo teste Scott-Knott $(p>0,05)$ e na linha pelo teste Tukey $(p>0,05)$.

PEREIRA et al. (2015) relatam que valores maiores de massa seca da parte aérea (14 dias) tem relação com aumento de produtividade de grãos, e ainda, ABREU et al. (2014) e SBRUSSI \& ZUCARELI (2015) consideram que sementes mais vigorosas apresentam maior tolerância ao estresse.

Os meio-irmãos P2, L7, L9 e L11 apresentaram plântulas com comprimento médio total e da parte aérea superiores, quando as sementes foram obtidas em cultivo sem nitrogênio em cobertura e colocadas para germinar sem o efeito de estresse salino (Tabela 3). No meio-irmão L8, foi observado o mesmo fato quando as condições foram $150 \mathrm{~kg} \mathrm{ha}^{-1}$ de $\mathrm{N}$ em cobertura e germinação sem efeito do estresse salino (Tabela 3).

Em condições de estresse salino na germinação, o meio-irmão L8 apresentou médias superiores de comprimento médio total $(15,3 \mathrm{~cm})$, radicular $(9,54 \mathrm{~cm})$ e da parte aérea $(5,75 \mathrm{~cm})$, e da massa seca

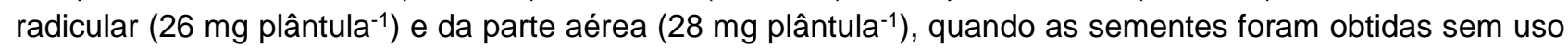
de nitrogênio em cobertura (Tabelas 4 e 5). Resultados semelhantes também foram observados no meioirmão P6, quanto ao comprimento médio total $(14,1 \mathrm{~cm})$ e radicular $(9,05 \mathrm{~cm})$, e na massa seca radicular (22 $\mathrm{mg}$ plântula $\left.{ }^{-1}\right)$ e da parte aérea (25 $\left.\mathrm{mg}_{\text {plântula }}{ }^{-1}\right)$.

Quando as sementes foram obtidas em condições de cultivo com o uso de N em cobertura $(150 \mathrm{~kg}$ ha $^{-1}$ ) e colocadas para germinar sob efeito do estresse salino, o meio-irmão L9 apresentou médias superiores de comprimento médio total $(25,3 \mathrm{~cm})$, radicular $(15,16 \mathrm{~cm})$ e da parte aérea $(10,09 \mathrm{~cm})$, e da massa seca radicular (31 $\mathrm{mg}$ plântula $\left.\mathrm{a}^{-1}\right)$ e da parte aérea (34 $\mathrm{mg}$ plântula $\left.\mathrm{a}^{-1}\right)$.

O meio-irmão $L 9$, ainda, foi o que apresentou influência significativa $(p<0,05)$ e positiva do uso do nitrogênio em cobertura em todos os atributos relacionados ao vigor, quando a germinação foi realizada com estresse salino (Tabelas 4 e 5). No entanto, a influência significativa do nitrogênio apresentou resultados diferentes em função do genótipo avaliado e das condições de germinação imposta às sementes. De acordo com GONDIM et al. (2006), o nitrogênio, como constituinte das proteínas, desempenha papel importante no desenvolvimento inicial do embrião durante a germinação. Entretanto, a resposta da planta ao $\mathrm{N}$ é complexa e controlada por vários genes/alelos que se expressam diferentemente nos seus órgãos (CAÑAS et al. 2011). 
Tabela 4. Comprimento médio total, radicular e da parte aérea $\left(\mathrm{C}_{\mathrm{T}}, \mathrm{C}_{\mathrm{R}}, \mathrm{C}_{\mathrm{PA}}\right.$, em $\left.\mathrm{cm}\right)$ das plântulas de 12 meio-irmãos de milho cultivados com dois níveis de nitrogênio em cobertura $\left(0 ; 150 \mathrm{~kg} \mathrm{ha}^{-1}\right)$, em condições de germinação com estresse salino. Tocantins, 2012/2013.

Table 4. Total, radicular and aerial part length $\left(\mathrm{C}_{\mathrm{T}}, \mathrm{C}_{\mathrm{R}}, \mathrm{CPA}_{\mathrm{PA}}\right.$ in $\left.\mathrm{cm}\right)$ of seedlings of the 12-maize half-sib families growing with two levels of covered nitrogen $\left(0 ; 150 \mathrm{~kg} \mathrm{ha}^{-1}\right)$, in germination conditions with saline stress. Tocantins, 2012/2013

\begin{tabular}{|c|c|c|c|c|c|c|c|c|c|c|c|c|}
\hline \multirow{3}{*}{$\begin{array}{l}\text { Meio- } \\
\text { irmãos } \\
\text { P1 }\end{array}$} & \multicolumn{4}{|c|}{$\mathrm{C}_{\mathrm{T}}$} & \multicolumn{4}{|c|}{$C_{R}$} & \multicolumn{4}{|c|}{$\mathrm{C}_{\mathrm{PA}}$} \\
\hline & \multicolumn{2}{|c|}{$0 \mathrm{~N}$} & \multicolumn{2}{|c|}{$150 \mathrm{~N}$} & \multicolumn{2}{|c|}{$0 \mathrm{~N}$} & \multicolumn{2}{|c|}{$150 \mathrm{~N}$} & \multicolumn{2}{|c|}{$0 \mathrm{~N}$} & \multicolumn{2}{|c|}{$150 \mathrm{~N}$} \\
\hline & 12,1 & $\mathrm{Aa}$ & 10,8 & $\mathrm{Ca}$ & 8,41 & $\mathrm{Aa}$ & 7,71 & $\mathrm{Ba}$ & 3,69 & $\mathrm{Ca}$ & 3,13 & $\mathrm{Da}$ \\
\hline P2 & 12,0 & $\mathrm{Aa}$ & 12,2 & $\mathrm{Ca}$ & 7,78 & $\mathrm{Ba}$ & 7,58 & $\mathrm{Ba}$ & 4,26 & $\mathrm{Ba}$ & 4,64 & $\mathrm{Ca}$ \\
\hline P3 & 12,2 & $\mathrm{Aa}$ & 14,2 & $\mathrm{Ba}$ & 7,66 & $\mathrm{Ba}$ & 8,15 & $\mathrm{Ba}$ & 4,58 & $\mathrm{Bb}$ & 6,03 & $\mathrm{Ba}$ \\
\hline P4 & 13,2 & $\mathrm{Aa}$ & 12,5 & $\mathrm{Ca}$ & 8,30 & $\mathrm{Aa}$ & 8,26 & $\mathrm{Ba}$ & 4,91 & $\mathrm{Ba}$ & 4,26 & $\mathrm{Ca}$ \\
\hline P5 & 10,7 & $\mathrm{Ba}$ & 12,6 & $\mathrm{Ca}$ & 7,46 & $\mathrm{Ba}$ & 7,96 & $\mathrm{Ba}$ & 3,21 & $\mathrm{Cb}$ & 4,59 & $\mathrm{Ca}$ \\
\hline P6 & 14,1 & $\mathrm{Aa}$ & 12,2 & $\mathrm{Ca}$ & 9,05 & $\mathrm{Aa}$ & 7,99 & $\mathrm{Ba}$ & 5,06 & $\mathrm{Ba}$ & 4,24 & $\mathrm{Ca}$ \\
\hline L7 & 10,1 & $\mathrm{Ba}$ & 10,4 & $\mathrm{Ca}$ & 6,90 & $\mathrm{Ba}$ & 6,91 & $\mathrm{Ba}$ & 3,20 & $\mathrm{Ca}$ & 3,51 & $\mathrm{Da}$ \\
\hline L8 & 15,3 & $\mathrm{Aa}$ & 13,5 & $\mathrm{Ba}$ & 9,54 & $\mathrm{Aa}$ & 8,81 & $\mathrm{Ba}$ & 5,75 & $\mathrm{Aa}$ & 4,71 & $\mathrm{Cb}$ \\
\hline L9 & 13,1 & $A b$ & 25,3 & $\mathrm{Aa}$ & 8,45 & $A b$ & 15,16 & $\mathrm{Aa}$ & 4,60 & $\mathrm{Bb}$ & 10,09 & $\mathrm{Aa}$ \\
\hline L10 & 13,3 & $\mathrm{Aa}$ & 14,0 & $\mathrm{Ba}$ & 7,56 & $\mathrm{Ba}$ & 8,61 & $\mathrm{Ba}$ & 5,74 & $\mathrm{Aa}$ & 5,43 & $\mathrm{Ba}$ \\
\hline L11 & 9,3 & $\mathrm{Bb}$ & 11,9 & $\mathrm{Ca}$ & 5,94 & $\mathrm{Ba}$ & 7,53 & $\mathrm{Ba}$ & 3,39 & $\mathrm{Cb}$ & 4,36 & $\mathrm{Ca}$ \\
\hline L12 & 9,9 & $\mathrm{Bb}$ & 14,4 & $\mathrm{Ba}$ & 7,04 & $\mathrm{Bb}$ & 8,95 & $\mathrm{Ba}$ & 2,81 & $\mathrm{Cb}$ & 5,45 & $\mathrm{Ba}$ \\
\hline
\end{tabular}

Médias com mesma letra pertencem ao mesmo grupo estatístico, na coluna pelo teste Scott-Knott $(p>0,05)$ e na linha pelo teste Tukey $(p>0,05)$.

Tabela 5. Massa seca radicular e da parte aérea $\left(\mathrm{MS}_{\mathrm{R}}, \mathrm{MS}_{\mathrm{PA}}\right.$, em mg plântula $\left.{ }^{-1}\right)$ das plântulas de 12 meioirmãos de milho cultivados com dois níveis de nitrogênio em cobertura $\left(0 ; 150 \mathrm{~kg} \mathrm{ha}^{-1}\right)$, em condições de germinação com ou sem estresse salino. Tocantins, 2012/2013.

Table 5. Radicular and aerial part dry weight ( $\mathrm{MS}_{\mathrm{R}}, \mathrm{MS}_{\mathrm{PA}}$, in $\mathrm{mg}$ seedling-1) of seedlings of the 12-maize half-sib families growing with two levels of covered nitrogen $\left(0 ; 150 \mathrm{~kg} \mathrm{ha}^{-1}\right)$, in germination conditions with and without saline stress. Tocantins, 2012/2013.

\begin{tabular}{|c|c|c|c|c|c|c|c|c|c|c|c|c|c|c|c|c|}
\hline \multirow{3}{*}{$\begin{array}{l}\text { Meio- } \\
\text { irmãos }\end{array}$} & \multicolumn{8}{|c|}{ Com estresse salino } & \multicolumn{8}{|c|}{ Sem estresse salino } \\
\hline & \multicolumn{4}{|c|}{$\mathrm{MS}_{\mathrm{R}}$} & \multicolumn{4}{|c|}{ MSPA } & \multicolumn{4}{|c|}{$\mathrm{MS}_{\mathrm{R}}$} & \multicolumn{4}{|c|}{ MSPA } \\
\hline & \multicolumn{2}{|r|}{ ON } & \multicolumn{2}{|c|}{$150 \mathrm{~N}$} & \multicolumn{2}{|c|}{ ON } & \multicolumn{2}{|c|}{$150 \mathrm{~N}$} & \multicolumn{2}{|c|}{ ON } & \multicolumn{2}{|c|}{$150 \mathrm{~N}$} & \multicolumn{2}{|r|}{$\mathrm{ON}$} & \multicolumn{2}{|c|}{$150 \mathrm{~N}$} \\
\hline & & & & & & & & $\mathrm{mg} \mathrm{f}$ & $\mathrm{la}^{-1}$ & & & & & & --- & \\
\hline P1 & 18 & $\mathrm{Ba}$ & 10 & $\mathrm{Cb}$ & 14 & $\mathrm{Ba}$ & 8 & $\mathrm{Ca}$ & 23 & $\mathrm{Ba}$ & 25 & $\mathrm{Ba}$ & 28 & $\mathrm{Ca}$ & 32 & $\mathrm{Da}$ \\
\hline P2 & 13 & $\mathrm{Ca}$ & 14 & $\mathrm{Ca}$ & 9 & $\mathrm{Ba}$ & 15 & $\mathrm{Ba}$ & 29 & $\mathrm{Aa}$ & 28 & $\mathrm{Ba}$ & 32 & $\mathrm{Ca}$ & 34 & $\mathrm{Da}$ \\
\hline P3 & 19 & $\mathrm{Ba}$ & 21 & $\mathrm{Ba}$ & 23 & $\mathrm{Aa}$ & 28 & $\mathrm{Aa}$ & 35 & $\mathrm{Aa}$ & 28 & $\mathrm{Ba}$ & 36 & $\mathrm{Ba}$ & 36 & $\mathrm{Da}$ \\
\hline P4 & 17 & $\mathrm{Ba}$ & 18 & $\mathrm{Ba}$ & 21 & $\mathrm{Aa}$ & 19 & $\mathrm{Ba}$ & 34 & $\mathrm{Aa}$ & 39 & $\mathrm{Aa}$ & 36 & $\mathrm{Ba}$ & 38 & $\mathrm{Ca}$ \\
\hline P5 & 15 & $\mathrm{Ca}$ & 11 & $\mathrm{Ca}$ & 16 & $\mathrm{Ba}$ & 18 & $\mathrm{Ba}$ & 32 & $\mathrm{Aa}$ & 38 & $\mathrm{Aa}$ & 39 & $\mathrm{Ba}$ & 43 & $\mathrm{Ba}$ \\
\hline P6 & 22 & $\mathrm{Aa}$ & 21 & $\mathrm{Ba}$ & 25 & $\mathrm{Aa}$ & 20 & $\mathrm{Ba}$ & 40 & $\mathrm{Aa}$ & 36 & $\mathrm{Aa}$ & 52 & $\mathrm{Aa}$ & 51 & $\mathrm{Aa}$ \\
\hline L7 & 19 & $\mathrm{Ba}$ & 20 & $\mathrm{Ba}$ & 16 & $\mathrm{Ba}$ & 15 & $\mathrm{Ba}$ & 34 & $\mathrm{Aa}$ & 36 & $\mathrm{Aa}$ & 40 & $\mathrm{Ba}$ & 40 & $\mathrm{Ca}$ \\
\hline L8 & 26 & $\mathrm{Aa}$ & 29 & $\mathrm{Aa}$ & 28 & $\mathrm{Aa}$ & 18 & $\mathrm{Bb}$ & 23 & $\mathrm{Ba}$ & 30 & $\mathrm{Ba}$ & 41 & $\mathrm{Ba}$ & 42 & $\mathrm{Ba}$ \\
\hline L9 & 19 & $\mathrm{Bb}$ & 31 & $\mathrm{Aa}$ & 19 & $A b$ & 34 & $\mathrm{Aa}$ & 32 & $\mathrm{Aa}$ & 31 & $\mathrm{Ba}$ & 41 & $\mathrm{Ba}$ & 37 & $\mathrm{Ca}$ \\
\hline L10 & 18 & $\mathrm{Ba}$ & 21 & $\mathrm{Ba}$ & 23 & $\mathrm{Aa}$ & 20 & $\mathrm{Ba}$ & 23 & $\mathrm{Ba}$ & 25 & $\mathrm{Ba}$ & 36 & $\mathrm{Ba}$ & 39 & $\mathrm{Ca}$ \\
\hline L11 & 13 & $\mathrm{Cb}$ & 18 & $\mathrm{Ba}$ & 21 & $A b$ & 31 & $\mathrm{Aa}$ & 25 & $\mathrm{Ba}$ & 30 & $\mathrm{Ba}$ & 41 & $\mathrm{Ba}$ & 44 & $\mathrm{Ba}$ \\
\hline L12 & 18 & $\mathrm{Ba}$ & 22 & $\mathrm{Ba}$ & 18 & $\mathrm{Ba}$ & 20 & $\mathrm{Ba}$ & 32 & $\mathrm{Aa}$ & 27 & $\mathrm{Ba}$ & 37 & $\mathrm{Ba}$ & 40 & $\mathrm{Ca}$ \\
\hline
\end{tabular}

Médias com mesma letra pertencem ao mesmo grupo estatístico, na coluna pelo teste Scott-Knott $(p>0,05)$ e na linha pelo teste Tukey $(p>0,05)$.

Resultados inconsistentes entre genótipos de milho, quanto à qualidade fisiológica da semente, também foram observados por GONDIM et al. (2006) e ZUCARELI et al. (2012), na avaliação do efeito de doses de N, e por OLIVEIRA et al. (2013), relacionado a diferentes tamanhos de semente. MOTERLE et al. (2006) observaram comportamento diferente dos cultivares de milho sob estresse salino, com grande variabilidade de resultados quanto ao comprimento de raiz primária e de parte aérea das plântulas. 
NERLING et al. (2013) afirmam que o genótipo exerce influência na tolerância ao estresse na germinação, e isso pode ter relação com o fato de que a qualidade fisiológica é determinada ou adquirida no desenvolvimento da semente (ABREU et al. 2014). Assim, como a resposta ao estresse depende da qualidade fisiológica (ÁVILA et al. 2007), é fundamental e importante a escolha do genótipo adequado (GONDIM et al. 2006, MOTERLE et al. 2006).

Os meio-irmãos P6 e L8 foram aqueles que se destacaram entre os demais, quanto à qualidade fisiológica da semente, quando em condições de estresse, tanto no cultivo quanto na germinação. Estes podem ser utilizados como fonte de germoplasma para o melhoramento genético, como o meio-irmão L9 com relação à resposta positiva à aplicação do $\mathrm{N}$ durante o cultivo.

\section{CONCLUSÃO}

A concentração de $16,73 \mathrm{~g} \mathrm{~L}^{-1}$ de $\mathrm{KCl}$ promoveu reduções significativas na germinação e vigor de sementes de meio-irmãos de milho, porém, permitiu a visualização do efeito da adubação nitrogenada em cobertura, bem como o efeito de genótipo. O meio-irmão L9 foi o mais responsivo à utilização de $\mathrm{N}$ em cobertura quanto à qualidade fisiológica da semente, no entanto, os demais meio-irmãos apresentaram respostas inconsistentes. Em condições sem uso do $\mathrm{N}$ em cobertura para obtenção de sementes e estresse salino na germinação, destacaram-se os meio-irmãos P6 e L8.

\section{REFERÊNCIAS}

ABREU VM et al. 2014. Physiological performance and expression of isozymes in maize seeds subjected to water stress. Journal of Seed Science 36: 40-47.

ALLARD RW \& BRADSHAW AD. 1964. Implications of genotype-environmental interactions in applied plant breeding. Crop Science 4: 503-508.

ÁVILA MR et al. 2007. Teste de comprimento de plântulas sob estresse hídrico na avaliação do potencial fisiológico das sementes de milho. Revista Brasileira de Sementes 29: 117-124.

BRASIL. 2009. Ministério da Agricultura, Pecuária e Abastecimento. Regras para análise de sementes. Brasília, DF: MAPA/ACS. 399p.

CAÑAS RA et al. 2011. An integrated statistical analysis of the genetic variability of nitrogen metabolism in the ear of three maize inbred lines (Zea mays L.). Journal of Experimental Botany 62: 2309-2318.

CARVALHO HWL et al. 2000. Avaliação de progênies de meios-irmãos da população de milho CMS-453 no Nordeste brasileiro. Pesquisa Agropecuária Brasileira 35: 1577-1584.

CARVALHO HWL \& SOUZA EM. 2007. Ciclos de seleção de progênies de meios-irmãos do milho BR 5011 Sertanejo. Pesquisa Agropecuária Brasileira 42: 803-809.

CHIMONYO VGP et al. 2014. Genotype x environment interactions and yield stability of stress-tolerant open-pollinated maize varieties in the Eastern Cape province, South Africa. South African Journal of Plant and Soil 31: 61-68.

COLOMBO GA et al. 2014. Potencial genético de famílias de meios-irmãos de milho pipoca para as condições de cerrado do sul do Tocantins. Comunicata Scientiae 5: 259-266.

CONUS LA et al. 2009. Germinação de sementes e vigor de plântulas de milho submetidas ao estresse salino induzido por diferentes sais. Revista Brasileira de Sementes 31: 67-74.

DIAS MCLL \& BARROS ASR. 1995. Avaliação da qualidade de sementes de milho. Londrina: IAPAR. 43p. (IAPAR. Circular, 88).

DUETE RRC et al. 2008. Manejo da adubação nitrogenada e utilização do nitrogênio ( $\left.{ }^{15} \mathrm{~N}\right)$ pelo milho em Latossolo Vermelho. Revista Brasileira de Ciência do Solo 32: 161-171.

FERREIRA ACB et al. 2001. Características agronômicas e nutricionais do milho adubado com nitrogênio, molibdênio e zinco. Scientia Agricola 58: 131-138.

GONDIM TCO et al. 2006. Avaliação da qualidade fisiológica de sementes de milho-crioulo sob estresse causado por baixo nível de nitrogênio. Revista Ceres 53: 413-417.

GRZYBOWSKI CRS et al. 2015. Testes de estresse na avaliação do vigor de sementes de milho. Revista Ciência Agronômica 46: 590-596.

MARSCHNER H. 1995. Mineral nutrition of higher plant. 2.ed. New York: Academic Press. 889p.

MOTERLE LM et al. 2006. Germinação de sementes e crescimento de plântulas de cultivares de milho-pipoca submetidas ao estresse hídrico e salino. Revista Brasileira de Sementes 28: 169-176.

NAKAGAWA J. 1999. Testes de vigor baseados no desempenho das plântulas. In: KRZYZANOSKI FC et al. (Ed.). Vigor de sementes: conceitos e testes. Londrina: ABRATES. p. 1-24.

NERLING D et al. 2013. Genetic diversity for physiological quality of seeds from corn (Zea mays L.) intervarietal crossbreeds. Journal of Seed Science 35: 449-456.

NERLING D et al. 2014. Qualidade física e fisiológica de sementes de milho durante o beneficiamento. Revista de Ciências Agroveterinárias 13: 238-246.

OLIVEIRA GE et al. 2013. Physiological quality and amylase enzyme expression in maize seeds. Ciência e Agrotecnologia 37: 40-48. 
PEREIRA LM et al. 2015. Seed quality, chlorophyll content index and leaf nitrogen levels in maize inoculated with Azospirillum brasilense. Revista Ciência Agronômica 46: 630-637.

RIBEIRO AC et al. 1999. Recomendações para o uso de corretivos e fertilizantes em Minas Gerais: $5^{\mathrm{a}}$ aproximação. Viçosa: Comissão de Fertilidade do solo do Estado de Minas Gerais. 359p.

ROTILI EA et al. 2015. Características pré e pós maturação fisiológica na divergência genética em milho sob diferentes níveis de nitrogênio. Enciclopédia Biosfera 11: 2940-2949.

SBRUSSI CAG \& ZUCARELI C. 2015. Germinação sob altas temperaturas para avaliação do potencial fisiológico de sementes de milho. Ciência Rural 45: 1736-1741.

TAIZ L \& ZEIGER E. 2009. Fisiologia vegetal. 4.ed. Porto Alegre: Artmed. 820p.

TIMÓTEO TS \& MARCOS FILHO J. 2013. Seed performance of different corn genotypes during storage. Journal of Seed Science 35: 207-215.

TONEL FR et al. 2013. Salicylic acid: physiological and biochemical changes in seeds and maize seedlings subjected to salt stress. Journal of Seed Science 35: 457-465.

ZUCARELI C et al. 2012. Doses e épocas de aplicação de nitrogênio em cobertura na qualidade fisiológica de sementes de milho doce. Revista Brasileira de Sementes 34: 480-487. 